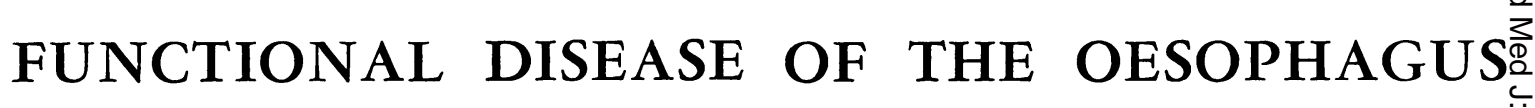

\author{
Ronald BeLSEY, M.S., F.R.C.S. \\ Surgeon-in-Charge, Thoracic Unit, Frenchay Hospital, Bristol; Consultant in Thoracic Surgery \\ to the South West Region
}

This term is used to embrace all those causes of dysphagia dependant upon a disorganization of the normal, co-ordinated, neuro-muscular function of the csophagus. All forms of dysphagia due to organic stenosis of the gullet, obstruction of its lumen by foreign bodies, or compression by extrinsic pressure are excluded.

Consideration of the causes of functional dysphagia is complicated by a lack of certainty in our knowledge of the normal activity of the gullet. This organ is not a mere inert drainpipe or passive conduit. Not only does it function with efficiency and consistency as a pump to propel the bolus or fluid from mouth to stomach under normal conditions in man, but can also overcome the forces of gravity, as demonstrated by the sportsman who, for a wager, imbibes a pint of beer whilst standing on his hands in the inverted position. Anyone who has witnessed a giraffe in the act of drinking will appreciate the dynamic propensities of this organ.

Peristalsis, as normally accepted, probably does not occur in the cesophagus.

The œsophagus is essentially a muscular tube with a sphincteric mechanism at both ends. The lumen normally contains a small quantity of saliva, and a larger quantity of air under a mild negative pressure reflecting and varying with the negative pressure in the pleural cavities. The sphincter at the upper end is controlled by the cricopharyngeal muscle in a state of tonic contraction. The vigilance of this sphincter is testified by the infrequency with which sudden inversion is accompanied by regurgitation of cesophageal contents. Were it not so, then every patient with achalasia of cardia and a resting cesophageal residue of up to two pints, or more, of fermenting food would run the risk of drowning every night. During normal deglutition the upper sphincter relaxes as the pharyngeal muscles voluntarily propel the bolus from the mouth. The lower sphincter is more complex. There is considerable doubt as to whether any intrinsic sphincter mechanism exists. Probably the control of gastroœsophageal reflux is maintained by a combination of factors: the pinch-cock action of the diaphragmatic hiatus on the lower osophagus; the angula- tion of the œsophago-gastric junction by the muscular sling of the right crus analogous to the action of the pubo-rectalis muscle on the ano- $\vec{\omega}$ rectal junction; the compression of the intraabdominal segment of cesophagus by positiveo pressure; the plugging of the lumen by valvular folds of gastric mucosa. Whatever its true nature, the lower sphincter shows a diminished sense of $f$ responsibility as compared with the upper.

During deglutition the bolus is first propelled î across the relaxed upper sphincter by the pharyngeal muscles. The sphincter then closes and a generalized contraction of the whole gullet ${ }_{3}^{-}$ rapidly moves the bolus onward into the stomach across the lower sphincter, after a temporary pause in the lower third of the organ. On screeniggo no true peristalsis is observed but repeated. ${ }^{\circ}$ rhythmical contractions of the gullet propel atlo but a small residue of the remaining bolus onwards. Serial pressure recordings from small balloons located at various points in the lumen have contributed to our knowledge of normal and $\stackrel{\Phi}{\Phi}$ abnormal function. As revealed by this method $\overrightarrow{\hat{F}}$ the contraction of the organ is progressive from 3 above downwards, although the rise in luminal? pressure during normal swallowing is probablyo generalized throughout the organ.

Disorganization of normal activity can occur 3 in several ways. The upper sphincter may failo to relax, with a consequent and very considerable . rise in pharyngeal pressure. Contraction rings, $\frac{O}{2}$ or segmentation, may occur at certain constant points in the cesophagus, replacing the normalo progressive contraction, and giving rise to the $D$ radiological appearances known as corkscrew․․ œsophagus. The degree of contraction at theN site of the rings may lead to complete occlusion at these points, with localized areas of raised 0 intra-luminal pressure. A bizarre sequel to this $\mathrm{N}$ rise of intra-luminal pressure is the development of 'blow-outs', or pulsion diverticula of mucosac through the muscular wall of the organ. Inter- $\mathbb{\Phi}$ mittent, but often severe, spasm of the lower end $\stackrel{\mathcal{P}}{+}$ of the gullet may occur as a result of gastro- $T$ œsophageal reflux and œsophagitis, less commonly from the swallowing of corrosive fluids. Impacted $\stackrel{\Phi}{\complement}$ foreign bodies can probably produce localized $\stackrel{\Phi}{\varrho}$ 
spasm at any point. Failure of the lower end of the gullet to relax during swallowing leads to the well-known condition of achalasia, with diffuse hypertrophy and dilatation of the whole organ.

Failure of the lower sphincter and the reflux of gastric secretion into the œsophagus is the essential functional disturbance responsible for most of the symptoms in hiatus hernia, and will not be considered further in this communication.

Finally, a rare form of dysfunction in which acute spasm of the mid-third of the organ accompanies the attempt to vomit large quantities of food and fluid after an alcoholic debauch, may lead to a rise in intra-luminal pressure sufficient to result in spontaneous rupture of the lower third with catastrophic results.

This communication is mainly concerned with the management of the three commoner forms of functional dysphagia amenable to surgical treatment-spasm of the upper sphincter, "corkscrew œsophagus', and achalasia of the cardia. The 132 cases reviewed were all admitted to a Regional Surgical Thoracic Unit under the care of one surgeon over a period of 20 years. The reason for investigation was either dysphagia or the pulmonary complications of œsophageal dysfunction. Non-surgical functional disorders, such as those due to basilar artery thrombosis or myasthenia gravis, will not be considered further although they complicate the problem of differential diagnosis.

\section{TABLE I}

Relative Frequency of Three Principal Forms OF FunCtional Disorder

(i) Upper cesophageal spasm, with pouch

$\begin{array}{llllll}\text { fii) } & \text {. } & \text {. } & \text {. } & & \\ \text { (ii) }\end{array}$

$\begin{array}{lllll}\text { (ii) Corkscrew osophagus } & \ldots & \ldots & \ldots & 18\end{array}$

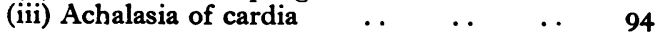

\section{Achalasia of Pharyngo-CEsophageal Junction with Pouch Formation}

Globus hystericus has been a popular diagnosis in any case where intermittent symptoms of dysphagia have been referred to the cervical cesophagus. In fact, the majority of these patients are suffering not from hysteria but from organic obstruction of the cervical œsophagus by muscular spasm, or achalasia, in the region of the pharyngoresophageal junction (Fig. I). The spasm may be sufficiently severe to lead to the formation of a pulsion diverticulum through the posterior wall of the pharynx. When the patient presents with the well-established picture of a pharyngeal diverticulum, the underlying obstructive element is usually overlooked, and completely ignored in planning treatment. This omission undoubtedly accounts for the unsatisfactory results of treatment and the high incidence of recurrent diverticulum

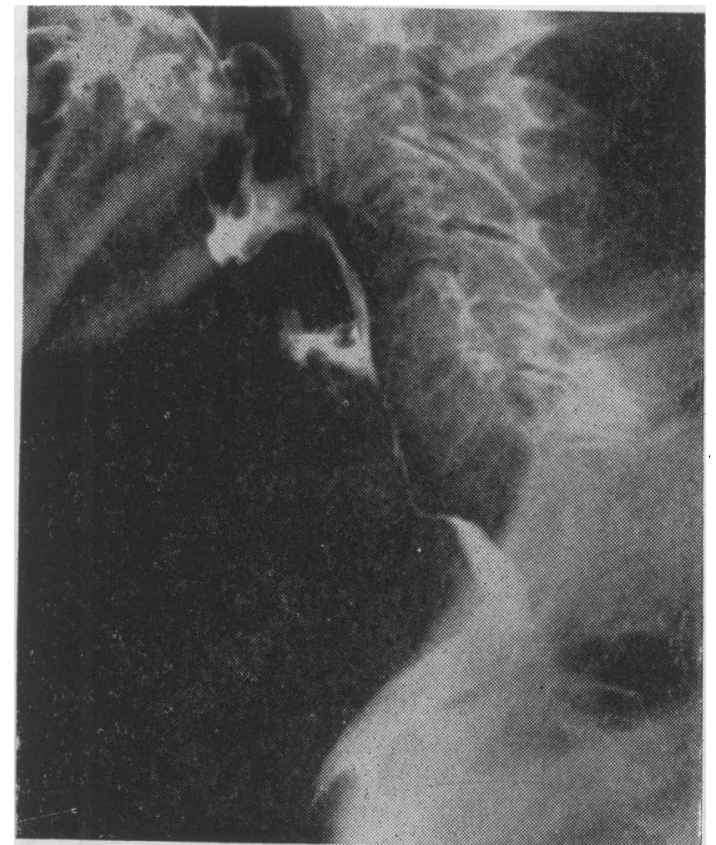

Fig. 1.-Severe spasm of the cervical œsophagus Dysphagia relieved by myotomy.

\section{1}

formation. Attention is diverted from the underlying cause of the dysphagia by the bizarre radiological manifestations of the sequelæ. The diagnosis is made largely on the symptoms, the localization of the obstruction, the radiological demonstration of a zone of persisting spasm in this area, and the exclusion of other possible causes of obstruction. A barium swallow examination may reveal a small pulsion diverticulum of the lower pharynx, too small to be able to contribute to the dysphagia. Esophagoscopy will contribute little to the diagnosis except to exclude other possible causes for the symptoms, e.g. a malignant stricture. Apart from the distress caused by the dysphagia, the importance of this lesion lies in the fact that it is the precursor and cause of a pharyngeal diverticulum. Before the onset of this complication the dysphagia is variable, and influenced by emotional stress-hence the term 'globus hystericus'. Once a pouch has developed and assumed a dependant position the symptoms increase in severity and are more constant. These symptoms are well recognized and will not be elaborated here, except to stress the frequency with which these patients present with chronic or recurring aspiration pneumonitis due to the nocturnal inhalation of the debris that may accumulate in the pouch.

The development of a pulsion diverticulum is a reversible process in its early stages, and relief of 


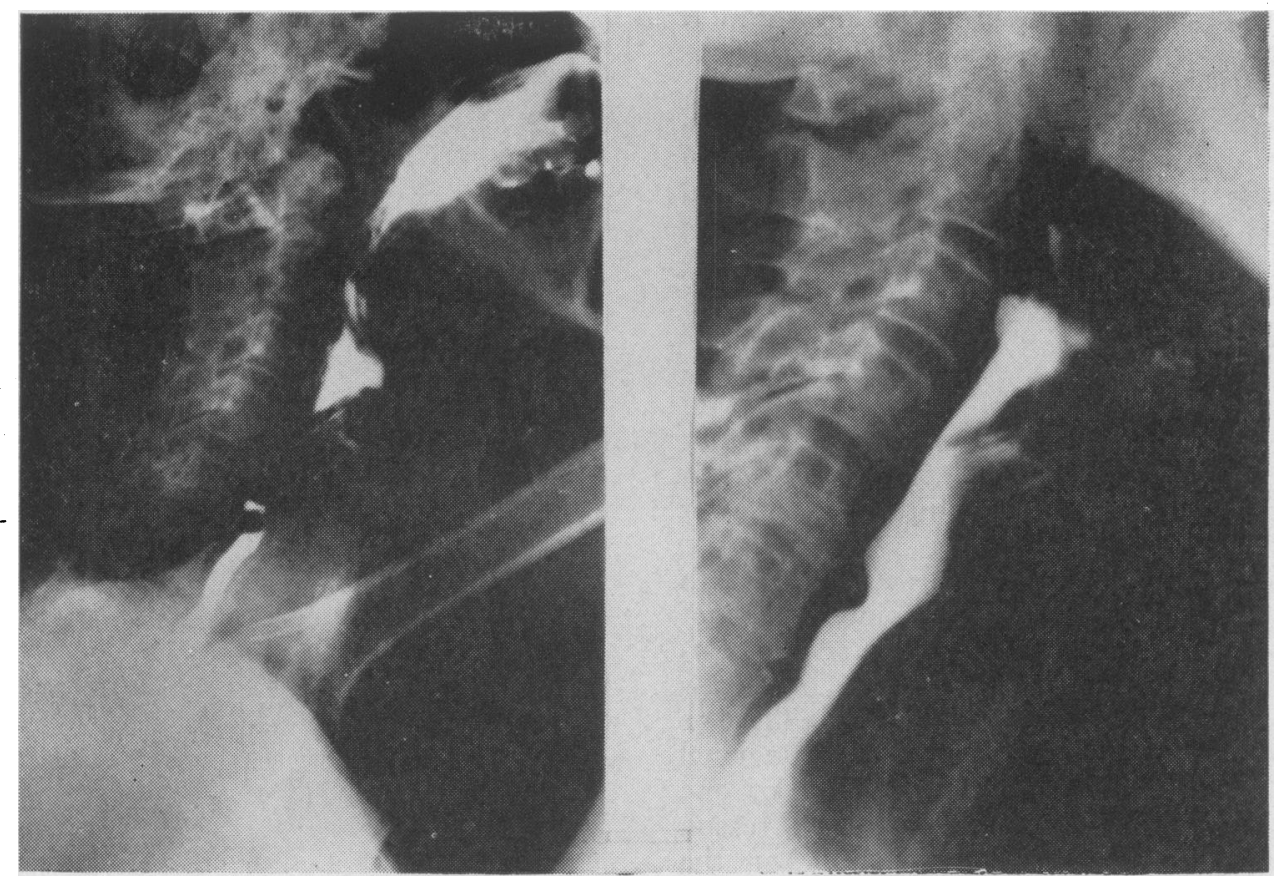

FIG. 2.-(a) Spasm of the cervical œesophagus with early pulsion diverticulum. (b) Following myotomy; the spasm and dysphagia have been relieved and the diverticulum has disappeared.

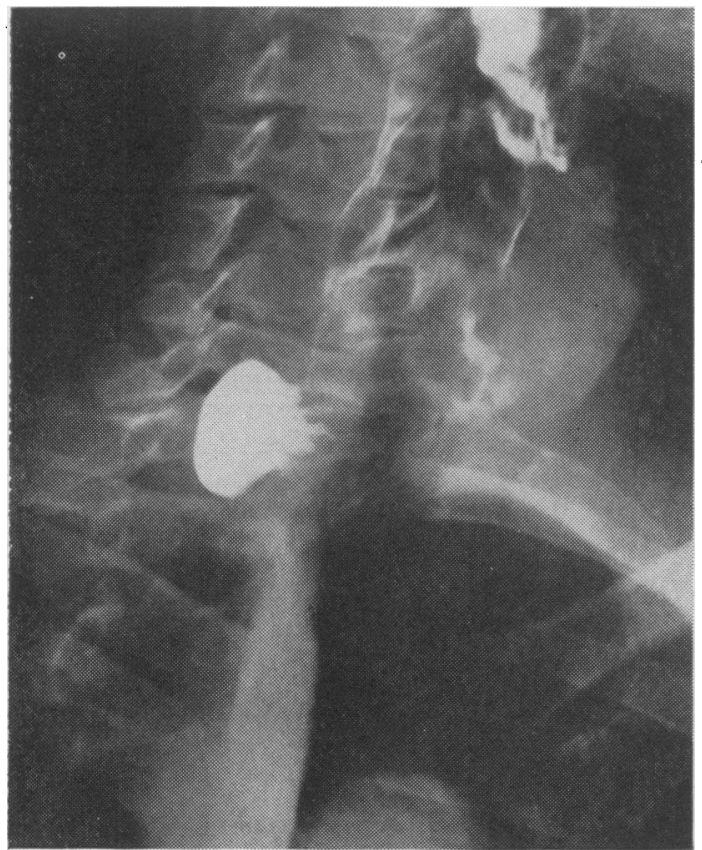

Fig. 3.-Pulsion diverticulum of the pharyngoœsophageal junction. The diverticulum disappeared completely following an upper œsophageal myotomy.

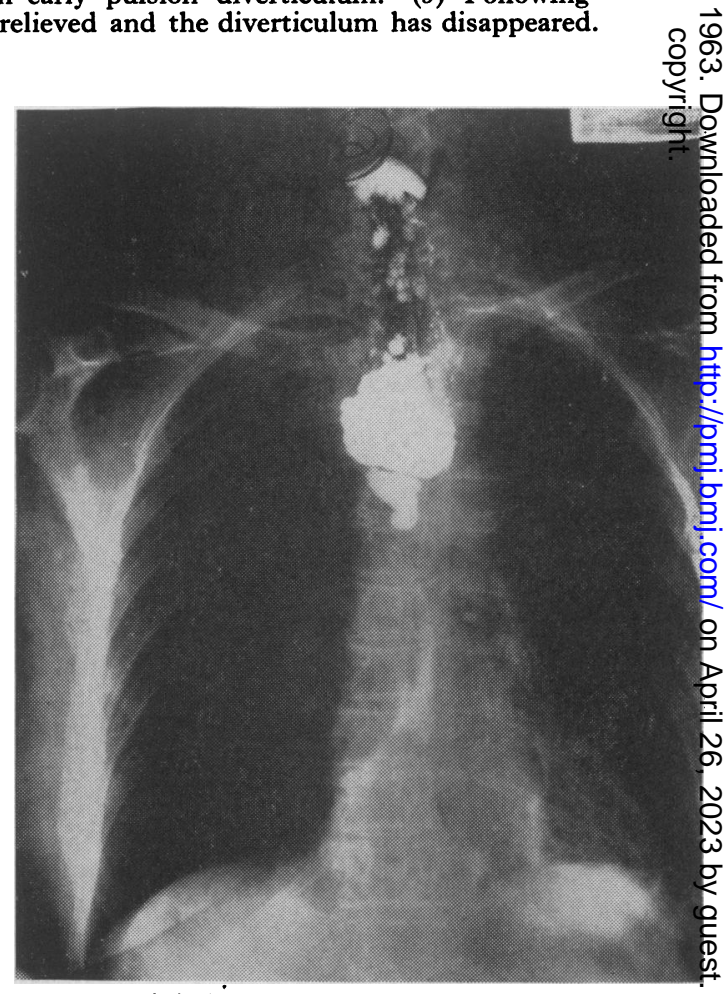

FIG. 4.-Lipiodol accumulating in the mediastinum following instrumental perforation of a pharyngea diverticulum. Spontaneous healing occurred, an $\Phi$ at a later date successful myotomy and diverticulo pexy were carried out. 


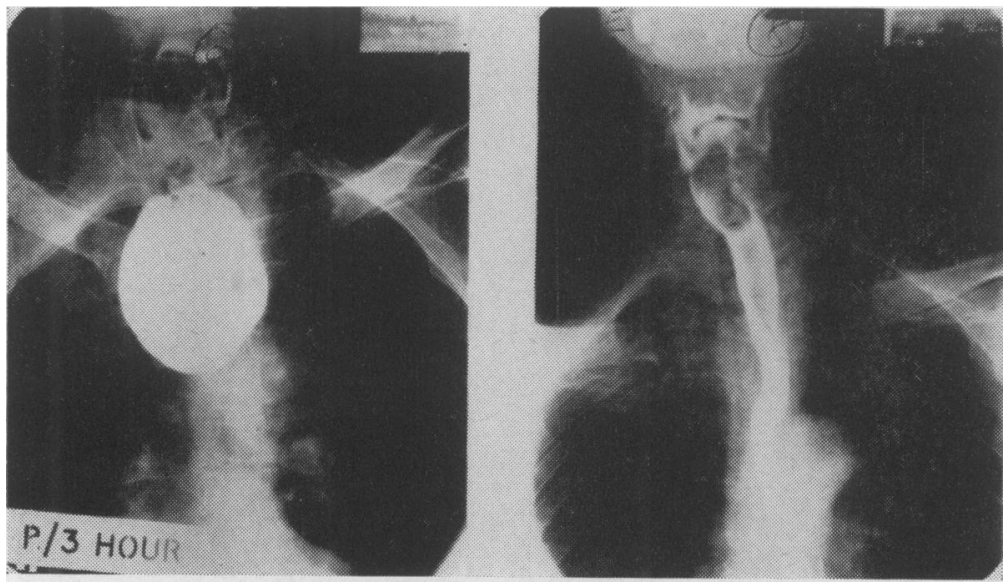

FIG. 5.-(a) Pharỳngeal diverticulum. œsophageal myotomy.

(b) Following diverticulopexy and

the obstruction may lead to rapid disappearance of the pouch (Fig. 2). Hence the importance of early recognition of this condition and its prompt relief.

Negus (1950) has advocated dilatation of the spastic pharyngo-œsophageal junction, repeated as necessary. Even in the hands of an expert, endoscopic dilatation of the osophagus is not devoid of risk of perforation or abrasion of the gullet, the risk being directly proportional to the frequency with which it has to be performed. If the dysphagia recurs after the initial dilatation, then other methods are indicated. That preferred by the author consists of an extramucous myotomy similar to the classical Heller operation performed on the lower œesophagus for the relief of achalasia. A vertical myotomy incision is made over the antero-lateral aspect of the lower half-inch of the pharynx and the upper two inches of the cervical cesophagus, usually on the right side. This procedure has proved satisfactory and has led to the disappearance of early diverticula (Fig. 3).

It is logical to assume that destruction of the superior œsophageal sphincter might expose the patient to the hazards of recurring aspiration pneumonitis. Provided that one of the csophageal sphincters remains competent, in practice the risk appears to be small, and no instance has been encountered so far in this small series. In fact there has been in this series no instance of 'œsophageal breathing' as anticipated by Negus (1950) in discussing the principles of this procedure.

Once a pulsion diverticulum larger than a grape has developed then dilatation becomes even more dangerous owing to the difficulty of gaining access to the lumen of the osophagus beside the neck of the pouch and the risk of perforating the

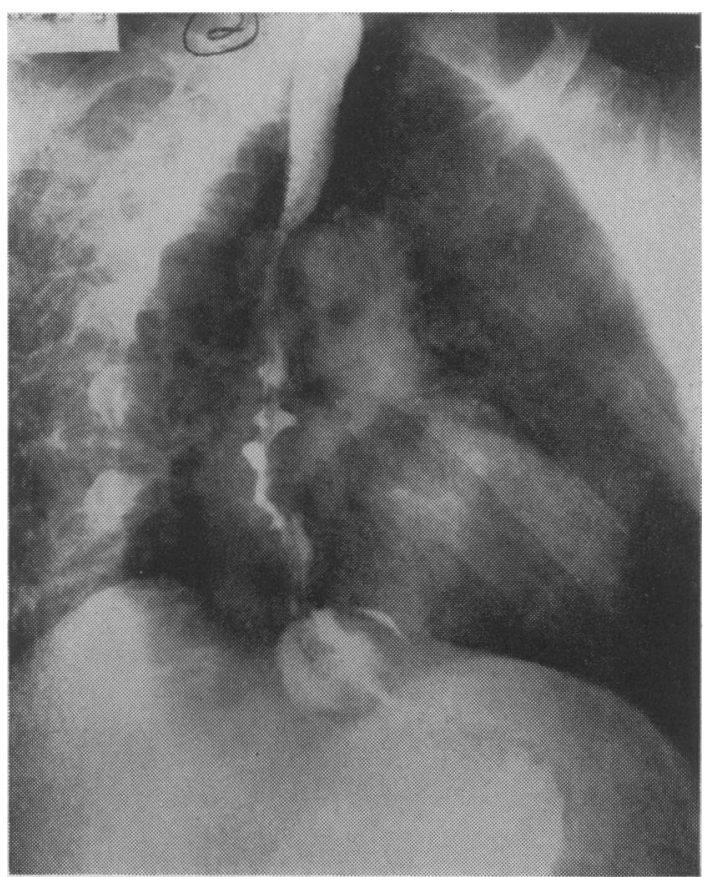

Fig. 6.-'Corkscrew cesophagus' associated with a Type I hiatus hernia.

bottom of the pouch and causing mediastinitis (Fig. 4). In addition to the myotomy already described, either excision of the pouch, or its inversion and suture to the anterior longitudinal ligament of the cervical spine in the up-ended position is necessary. The latter method has been used by the author in 18 cases with complete relief of symptoms and no recurrence of the pouch formation. It is preferred to excision 


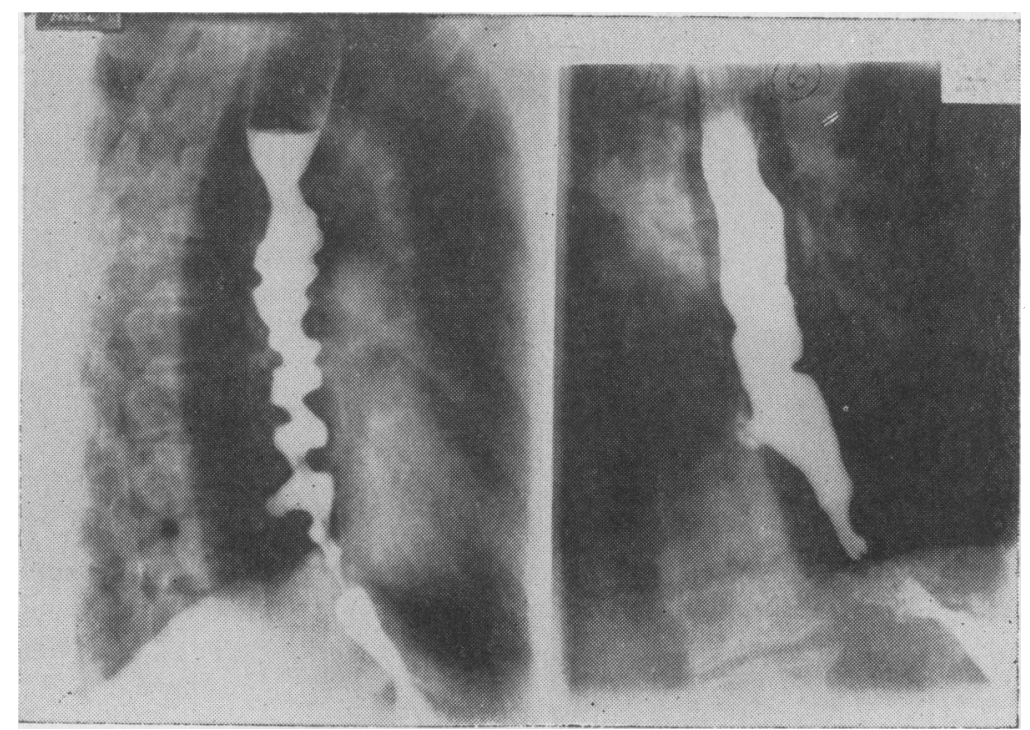

owing to the risk of a salivary fistula and the difficulty of performing a satisfactory repair of the thin posterior pharyngeal wall.

A direct attack on the pouch with no attempt to relieve the underlying cause will fail to relieve completely the dysphagia and will be followed by a high rate of recurrent pouch formation (Fig. 5). The longest follow-up period following myotomy and diverticulopexy has been ten years in this series. As yet there has been no recurrence of the pouch formation or dysphagia.

\section{Diffuse CEsophago-spasm}

This condition is commoner in the lower half of the csophagus. It is not peculiar to any particular age group or sex. It occurs in two forms-primary and secondary. The primary form appears to be closely allied to achalasia of the cardia in that examination of the osophagus reveals diffuse muscular hypertrophy indistinguishable from that which characterizes the latter condition. The secondary form is frequently associated with the presence of a hiatus hernia and gastro-œsophageal reflux (Fig. 6). The assumed association between the two is based upon the observation that surgical control of the gastrowophageal reflux leads to relief of the spastic condition of the gullet. The degree of muscular hypertrophy is less in the secondary form.

The radiological appearances presented by this condition are bizarre and have acquired the designation of 'corkscrew osophagus'. A series of contraction rings occurs intermittently through- out the lower half of the œsophagus. They have not been observed above the level of the aorti $\overline{6}$ arch. The rings always occur at the same level: and are not produced by an exaggerated form of peristalsis. Solitary or multiple pulsion diverticia are commonly associated with this form $\stackrel{*}{*}$ csophago-spasm and are undoubtedly 'blow-out\$ occurring in the zones of increased intra-luming pressure.

Much of our knowledge of the abnorma physiology of this condition has been elucidated by Good (working at the Mayo Clinic), by mean of serial pressure recordings from balloons located at various levels in the gullet. By this methog pressure changes at various points within the lumen can be recorded during swallowing and rest, and the disordered activity of the gullet corres lated with the symptoms. The commonest symptom of this condition is dysphagia, but in some cases the presenting symptom is chest pain of an anginal distribution. During swallowing the cons traction of the various spastic zones or rings may. occur in one of two ways: either synchronously or progressively from above downwards. In thee former instance the presenting symptom is dyst phagia; in the latter, substernal chest pain.

The indication for surgical treatment in this condition is the severity of the symptoms. The presence of pulsion diverticula in the lowep œsophagus rarely aggravates the symptoms and in contrast to those at the pharyngo-œsophagead junction, these do not need surgical treatmen except in the rare instances when they develop 


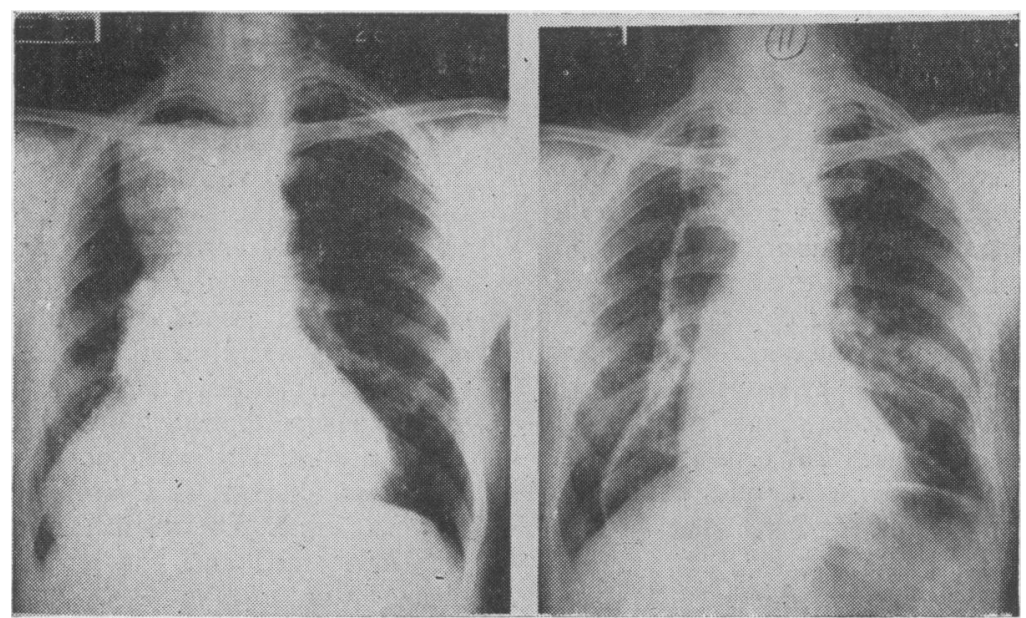

FIG. 8.-(a) Achalasia of the cardia complicated by gross œsophageal dilatation. Note fluid level above the right clavicle. (b) Two years following modified Heller's operation. Esophageal dilatation persists, but there is now no retention of fluid in the organ.

to a large size, and cause extrinsic pressure on the gullet below the level of the origin of the diverticulum (Fig. 7). Dilatation is rarely of value in this condition and if diverticula are present can be dangerous. Relief of the dysphagia can be achieved by a Heller type of myotomy performed on the lower œsophagus as in the treatment of achalasia of the cardia. A vertical extra-mucous myotomy incision is made over the lower three inches of œsophagus at least, and extended for a further half inch over the cardia of the stomach to ensure that all the circular muscle fibres of the lower œsophagus have been divided. If a hiatus hernia is present this should be repaired and a functioning valvular mechanism restored to the cardia to prevent any further gastro-œsophageal reflux. Minor degrees of œsophagospasm are frequently encountered complicating reflux and effective control of this reflux leads to prompt and complete relief of the spasm.

Excision of pulsion diverticula of the lower csophagus, on the mistaken assumption that they are responsible for the patient's symptoms, without relief of the functional obstruction will lead to catastrophic and often fatal complications in the form of broken-down suture lines and mediastinal and pleural suppuration.

\section{Achalasia of the Cardia}

The cause of this condition remains obscure. Commonly the obstruction is confined to the cardia but in the early stages of the condition there may be diffuse spasm of the lower half of the organ; in the later stages this is superseded by diffuse progressive dilatation and lengthening of the organ. The irregular spasm already described as 'corkscrew œsophagus' may be associated with achalasia and there appears to be a close pathological affinity between the two conditions. However, pulsion diverticula are rarely seen in achalasia. There is diffuse uniform hypertrophy of the muscle layers of the œsophagus, maximal in the lower half.

At the cardia, the point of obstruction, the architecture of the muscle layers suddenly becomes normal and in this region there is no hypertrophy, supporting the thesis that the obstruction is due to failure of normal relaxation rather than true spasm. Pathological changes have been observed in the nerve plexes in the muscle layers but whether these are primary or secondary, congenital or acquired, is not known; nor is their significance in the ætiology of this condition. In longstanding cases the degree of dilatation or mega-csophagus that develops is prodigious and this undoubtedly jeopardizes the functional result following surgical relief of the obstruction. Moderate dilatation will disappear following operation; gross dilatation will diminish but some degree will remain permanently (Fig. 8).

Considerable quantities of ingested food and fluid, some taken several days previously, are commonly retained in the dilated gullet. In the erect position a fluid level can be seen in the region of the clavicles on radiological examination. Conditions are ideal for alcoholic fermentation, a fact which may explain the unnatural euphoria enjoyed by many patients suffering from this condition. Of greater importance is the diffuse retention œsophagitis that may occur in advanced 
cases. The mucosa is then reddened and œdematous, and has a characteristic granular appearance, bleeding readily if touched. There is no resemblance to the esophagitis caused by gastroœsophageal reflux. The significance of the œsophagitis is twofold. First, it appears to be a definite pre-malignant condition; reference to this will be made later. Second, the presence of gross retention osophagitis is a contra-indication to radical surgical treatment and some preliminary form of drainage is necessary to control it before any form of Heller procedure is attempted, owing to the risk of perforation or spontaneous rupture of the softened œdematous mucosa whose texture can rightly be likened to that of the proverbial wet blotting paper.

\section{Diagnosis}

Although dysphagia is the presenting symptom in approximately $90 \%$ of cases, the patient may come under observation in other ways. The dysphagia is of such long duration, and so insidious in onset and progress that the patient may accept his disability as natural and inevitable. Only by asking leading questions can the clinician elicit a true picture of the patient's disability. These patients are often diagnosed as hysterical in the early stages before obvious dilatation of the gullet has occurred. Four patients with achalasia were referred to the author from mental hospitals with a diagnosis of chronic depression and 'an œsophageal abnormality'. On questioning it was learnt that the patients were depressed merely because they could not swallow. Relief of the dysphagia resulted in a dramatic psychiatric cure.

Pulmonary complications due to recurring aspiration pneumonitis, following regurgitation of œsophageal contents at night when the upper cesophageal sphincter is caught off its guard, are common, and may result in extensive, diffuse, pulmonary fibrosis. The dyspnoea and pulmonary symptoms then overshadow the dysphagia. It is surprising that more patients do not drown in their own csophageal residue. The youngest patient in the author's series, a boy of 8 years, was admitted to hospital with a diagnosis of bronchiectasis for further investigation. It was the noise caused by the aspiration of fluid into the trachea and bronchi at night that led to the correct diagnosis. As long ago as 1943 Hurst described four cases with respiratory complications following regurgitation, and a fifth, fatal, case of asphyxia.

Achalasia may be brought to light as the result of a routine mass miniature radiography examination. The opaque, fluid-filled, dilated osophagus presents many of the radiological appearances of a mediastinal tumour. When the dilatation is greatest in the aircontaining upper third of the organ, a diagnosis of lung cyst, lung abscess, of even pneumothorax may be made.

In one instance the patient was admitted to medical ward with a diagnosis of acute rheurmatoid arthritis. Examination suggested acut: pulmonary osteoarthropathy and further investiv gation confirmed the presence of achalasia of the cardia. The association of pulmonary osteo arthropathy and cesophageal disease is no recognized. In this case the retention œsophagitio was so severe that all mouth feeding was stoppea and a preliminary gastrostomy performed: withif 48 hours all pain and swelling had disappeared from the joints. One month later a Heller operatiog was performed and the gastrostomy allowed to close.

Investigation is carried out by means of radio logical examination and cesophagoscopy. Thẹ radiological appearances are well recognize $f$ especially the smooth, conical, constriction confined to the cardia that characteristicallo relaxes after the inhalation of octyl nitrite. The protean appearances presented by the dilated csophagus on plain radiological examinatio mimic all forms of mediastinal pathology. I接 no other form of œsophageal obstruction is comparable degree of cesophageal dilatation sere

Esophagoscopic examination is unsatisfactory owing to the difficulty of clearing the gullet obiti retained debris. The examination is safer wher performed in the sitting position under loc 太 anæsthesia; induction of general anæsthesia may lead to sudden flooding of. the air passages with œsophageal contents. The main object of th $\vec{B}$ examination is to inspect the mucosa and determin the severity of the retention œsophagitis. careful inspection is carried out for any signs of early malignancy. It is rare for any convincing view to be obtained of the cardia and the exclusion of a malignant or benign stricture at the card may be impossible.

\section{Relationship to Carcinoma of the Esophagus}

In the author's series of 94 cases of achalasia of the cardia there were eight cases of œsophage carcinoma. In four cases the patients presented with severe obstruction due to advanced carcings mata; radiological examination revealed the underlying achalasia and megacsophagus. The remaining four patients had previously beefr treated for achalasia and returned with recurrent obstruction due to malignant strictures 15 montl to five years following the Heller operation. In a eight cases the growths were squamous cell in type, were situated in the mid-third of the gullet and had reached an advanced stage before obstrue tive symptoms occurred owing to the previous 


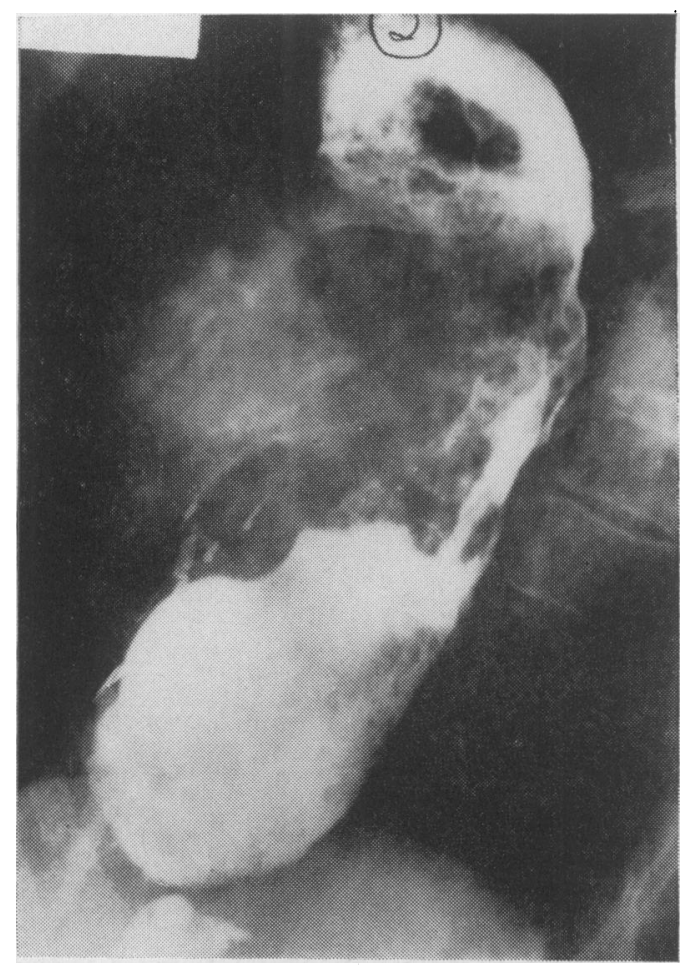

Fig. 9.-Achalasia of the card a complicated by an advanced carcinoma of the middle third of the œsophagus.

dilatation of the œsophagus (Fig. 9). In one case multiple carcinomata were present. In aseriesofover 500 cases of hiatus hernia complicated by reflux œsophagitis observed and treated by the author, there was no instance of a squamous cell carcinoma complicating the œsophagitis although several co-existing adenocarcinomata of the cardia were encountered. This evidence suggests that the retention œsophagitis complicating achalasia is a pre-malignant condition involving a high risk of malignant degeneration; that the reflux œsophagitis found in association with a hiatus hernia is not a pre-malignant condition. Of great interest are the four patients who developed malignant strictures subsequent to Heller operations, and in one case five years later. It is probable in retrospect that malignant degeneration had already commenced at the time of the original operation. As already stated it is notoriously difficult to conduct a thorough examination of the whole œsophageal mucosa, and when grossly inflamed and obscured by rotting food debris and barium an early carcinoma can easily escape detection. In four of the cases determined but ultimately futile attempts were made to resect and reconstruct the gullet, but in no instance was the growth operable by normal standards. It is logical to assume that the sooner steps are taken to relieve the obstruction and œsophagitis in achalasia, the less the risk of subsequent malignant degeneration.

\section{Treatment}

Pre-operative care is important. If gross œsophagitis is present a course of octyl nitrite inhalations following low residue meals will drain the œsophagus from below. It may be necessary to pursue this regimen for three to four weeks before it is safe to proceed with surgical treatment. In two cases in the author's series it was necessary to perform temporary gastrostomies and stop all mouth feeding to bring the inflammation under control. Esophageal washouts are dangerous owing to the risk of drowning the patient.

An intensive course of physiotherapy to improve the pulmonary complications is usually called for. The patient's nutritional state may be severely depleted from chronic starvation; this again must be corrected and blood transfusion may be necessary.

Numerous operations have been described for the relief of this condition. Repeated dilatations enjoyed a short vogue of popularity which was not justified by the results, and has now been largely abandoned by even its most fervent advocates. Various forms of œsophago-gastrostomy have been performed, to be followed in a high percentage of cases by disastrous gastrocsophageal reflux and ulceration. An extramucous myotomy (Heller operation) dividing all the circular muscle fibres over the lower two to three inches of œsophagus is the most satisfactory operation available at the present time. The operation is best performed through the chest as only an inadequate myotomy incision is possible through the abdominal approach. Franklin has rightly said that it is an easy operation to perform but a difficult operation to perform well. In its original form the operation was not entirely successful, the occasional poor results being due to the development of fibrous strictures secondary to gastro-œsophageal reflux. It has not been generally recognized that any surgical interference with the region of the cardia may weaken the already precarious valvular mechanism, and precipitate the development of a hiatus hernia or patulous cardia and its sequelæ. The author has in recent years employed a modification of the Heller procedure. Recognizing the risk of a hiatus hernia developing, the cardia is boldly freed from its attachments and following the myotomy a formal hernia repair is carried out 


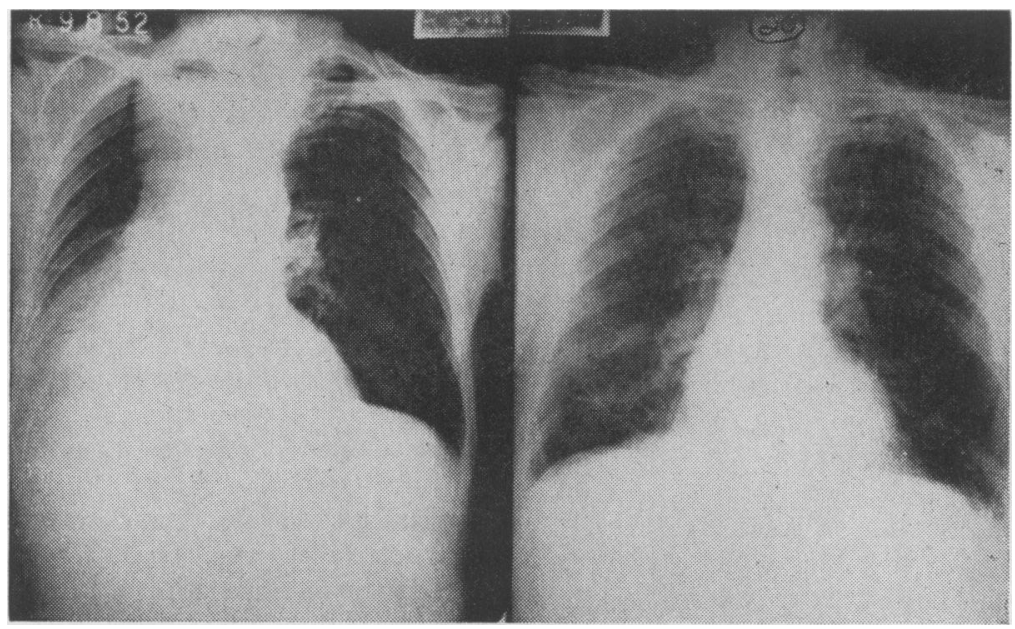

FIG. 10.-(a) Mega-csophagus complicating achalasia of the cardia. (b) Nine years following modified Heller myotomy; the cesophageal dilatation has disappeared.

by creating an acute angle of entry at the cardia, restoring an intra-abdominal segment of cesophagus, and approximating the two halves of the right crus behind the hiatus to form a buttress against which the intra-abdominal segment of cesophagus can be compressed by the abdominal pressure. In a personal series of 90 cases treated surgically, in the first 56 a formal Heller operation was carried out with satisfactory initial results, but in six cases, or $11 \%$, fibrous strictures subsequently developed of sufficient severity to need cesophago-gastric resection and reconstruction (Fig. 10). In the last 34 cases the modified operation has been performed and as yet there has been no instance of stricture formation or subsequent necessity for further surgery. There was one post-operative death in this series, from uræmia due to co-existing chronic nephritis.

The surgical relief of functional csophageal obstruction is justified by the gratitude of the patient, by the satisfactory long-term results, and by the relative safety of the surgical procedures indicated.

\section{Summary}

I. A series of 132 cases of functional disorders of the œsophagus has been described. Of these required surgical treatment for relief of dysphagia or the pulmonary complications of œsophagea obstruction.

2. No operation for pharyngeal diverticulum is complete unless the underlying spasm of th $\vec{G}$ upper sphincter is relieved.

3. Pulsion diverticula of the lower asophagus rarely need surgical treatment; the underlying. functional obstruction frequently does.

4. Achalasia of the cardia is a pre-malignan condition.

5. The Heller operation must be accompanied by the reconstruction of a functioning valvulae mechanism at the cardia to prevent subsequent gastro-œsophageal reflux and fibrous stenosis.

\section{REFERENCES}

FrankLIN, R. H.: Personal communication. Frankin, R. H.: Personal communication.
Hurst, SrR A. (r943): Respiratory Complications of Achalasia of the Cardia with Mega-Cesophagus, Guy's Hosp. Rep.
92, 68.

Nequs, V. E. (1950): Pharyngeal Diverticula: Observations on Their Evolution and Treatment, Brit. Y. Surg., 38, 129 NJ 